\title{
Effects of Wall Curvature on the Dynamics of an Impinging Jet and Resulting Heat Transfer
}

\author{
G.Camerlengo, D. Borello, A. Salvagni and J. Sesterhenn
}

\begin{abstract}
The effects of wall curvature on the dynamics of a round subsonic jet impinging on a concave surface are investigated for the first time by direct numerical solution of the compressible Navier-Stokes equations. Impinging jets on curved surfaces are of interest in several applications, such as the impingement cooling of gas turbine blades. The simulation is performed at Reynolds and Mach numbers respectively equal to 3,300 and 0.8 . The impingement wall is kept at a constant temperature, $80 \mathrm{~K}$ higher than that of the jet at the inlet. The nozzle-to-plate distance (measured along the jet axis) is set to $5 D$, with $D$ the nozzle diameter. In order to highlight the curvature effects, the present results are compared to a previous study of jet impinging on a flat plate. The specific influence of wall curvature is investigated through a frequency analysis based on discrete Fourier transform and dynamic mode decomposition. We found that the peak frequencies of the heat transfer also dominate the dynamics of primary vortices in the free jet region and secondary vortices produced by the interaction of primary vortices and the target plate. These frequencies are approximately $30 \%$ lower than those found in the reference study of impinging jet on a flat plate. Imperceptible differences were instead found in the time-averaged integral heat transfer.
\end{abstract}

Keywords Impinging jet $\cdot$ Curved surface $\cdot$ DNS $\cdot$ Heat transfer $\cdot$ DMD

\footnotetext{
G. Camerlengo $(\varangle) \cdot$ J. Sesterhenn

Institut für Strömungmechanik und Technische Akustik,

Technische Universität Berlin, Müller-Breslau-Str. 15, 10623 Berlin, Germany

e-mail: gabriele.camerlengo@tu-berlin.de

D. Borello · A. Salvagni

Dipartimento di Ingegneria Meccanica e Aerospaziale,

Università degli Studi di Roma "La Sapienza", Via Eudossiana 18, 00184 Roma, Italy

R. King (ed.), Active Flow and Combustion Control 2018,

Notes on Numerical Fluid Mechanics and Multidisciplinary Design 141,

https://doi.org/10.1007/978-3-319-98177-2_22
} 


\section{Introduction}

Impinging jets are employed as efficient cooling techniques in several applications. For instance, they are widely used for the cooling of gas turbines components, electronic parts and stock material during material forming processes. Compared to other heat transfer methods (without phase change), the impingement cooling offers efficient use of fluid. For example, in order to produce a given heat transfer coefficient, the flow rate required for the impingement cooling may be two order of magnitude smaller when compared with standard convective confined cooling ${ }^{1}$ [1]. In turbine applications, impingement jets are used to cool the combustor case, the combustor can, the turbine casing and the high temperature turbine blades. Specifically for the latter purpose, typical operative temperature differences may lead to required heat fluxes of the order $10^{6} \mathrm{~W} / \mathrm{m}^{2}$.

The mechanism of heat transfer associated to impinging jets is dominated by turbulence dynamics and the complete comprehension of the whole phenomenology is far to be reached, although, in view of the great interest in their applications, strong research efforts have been made. Moreover, curved cooled surfaced (such as, in turbomachinery applications, turbine vane and blade mid-chord regions) are often approximated as flat surfaces. Many studies are based on this configuration, and regardless of the flow properties (Reynolds number, Prandtl number and Mach number), some general features are shown. With focus on numerics, we recall, among others, the interesting works by [2, 3].

Cornaro et al. [4] analyzed the impingement jet flow on flat, concave and convex surfaces. Several tests were done by changing jet diameter, surface curvature, nozzleto-plate distance, Reynolds number and turbulence intensity. They observed that low turbulence intensity at the inlet favors the development of well-organized turbulent structures in the free jet that become more and more unstable when the inlet turbulence increases. Such structures generate axial velocity oscillations leading to accelerationdeceleration of the characteristic ring vortices, which have an axial distance that reduces when the Reynolds number increases. The presence of a concave surface made the flow more unstable when compared with convex or flat plate. In fact, the flow leaving the concave surface (here extending for about 210 to 240 degrees) interacts with the primary jet, disturbing the structures here present. As for the influence of the nozzle-to-plate distance, it is noted that, increasing such distance, stronger oscillations of the stagnation point occur, contributing to an earlier breakdown of the vortices reaching the solid surface. Finally, an increase of the relative curvature leads to fewer and less stable vortex structures.

Lee et al. [5] studied the heat transfer and the wall pressure coefficient profiles on a concave surface impacted by a fully developed jet. They found that the heat flux through the plate increases with surface curvature, due to the reduction of the boundary layer thickness and the development of more robust Taylor-Görtler vortices. They also observed a change in the correlation between heat flux and Reynolds

\footnotetext{
${ }^{1}$ Convective confined cooling occurs when heat is transferred within confined systems such as pipes, closed conduits and heat exchangers.
} 
number when the nozzle-to-plate distance becomes greater than 4 , i.e. the impact surface is outside of the potential core of the jet. This is due to the greater turbulence level of the impacting jet. They also showed that the secondary peak of the Nusselt number profile at the wall (see also [6]) cannot be observed with a nozzle-to-plate distance of 10 , whereas they are visible when this distance is set to 4 . In the latter case, the magnitude of the secondary peak increases with Reynolds number and surface curvature. At a Reynolds of 11,000 and with the minimum considered plate curvature, an inflection point appears in place of the secondary peak.

Choi et al. [7] measured mean velocity and velocity fluctuations for an impinging jet flow over a concave surface. They observed that the increase of the local heat transfer rate and the resulting secondary peak in the Nusselt number profile are related to the existence and strength of velocity fluctuations.

Gilard and Brizzi [8] carried out PIV measurements of the aerodynamic of a slot jet impinging on a concave wall. They focused on the influence of the curvature. They demonstrated that for low curvature, the flow exhibits three different alternating behaviors with large modifications of turbulent variables, indicating the occurrence of strong turbulent instabilities.

Recently, [9] investigated the dominant structures in an impinging jet flow on a concave surface by means of PIV measurements. Although the heat transfer was not directly measured, the authors related such structures to the r.m.s. velocity of the jet in order to extract useful information for the estimation of the position of the secondary peak in the Nusselt number profile at the wall. Results were shown in two perpendicular planes, parallel and perpendicular to the axis of the cylindrical surface. Higher value of the r.m.s. velocity profiles were measured along the curved surface when compared with the flat one, estimating therefore relevant differences in the position of the secondary peak along the considered planes.

Yang et al. [10] analyzed the effect of different nozzle exit on the heat transfer of a slot jet impinging on a concave surface. Although the nozzle-to-plate distance were too large to approximate the impingement cooling of a turbine leading edge, the authors showed that the effect of curvature becomes more prominent as the Reynolds number increases.

Aillaud et al. [11] performed a LES of a round jet impinging on a flat plate, investigating the link between the secondary peak in the Nusselt number distribution and near-wall vortices by means of statistical analysis (PDF, Skewness, and Kurtosis of heat transfer). They found that, where the secondary peak occurs, the wall structures produce a cold fluid flux towards the impingement plate.

As concerns numerical simulations of jets impinging on curved surfaces, very few studies are available in literature. Among these, it is worth mentioning the work by [12]. They carried out a zonal hybrid LES/RANS of flow and heat transfer for a round jet impinging on a concave hemispherical surface, mainly focusing on the assessment of the numerical methods. To the best knowledge of the authors of this paper, no direct numerical simulation (DNS) studies of the configuration under analysis so far exist in literature.

The objective of this DNS study, part of a more extensive research about internal cooling in gas turbines [13-15], is the analysis of the wall curvature effects on a round 
impinging jet flow, focusing on the modification of heat flux through the impingement plate. To this purpose, numerical results will be compared with a reference case of jet impinging on a flat plate [16-18]. Particularly, techniques devoted to capture the behavior of dynamic systems, such as discrete Fourier transform and dynamic mode decomposition [19], will be used.

\section{Computational Details}

\subsection{Numerical Methods}

The governing compressible Navier-Stokes equations are solved in the characteristic pressure-velocity-entropy formulation, as described by [20]. This formulation has particular advantages in the definition of boundary conditions and stability of the numerical solver. Since the smallest scales of turbulence are directly solved, no turbulence model is required. As concerns the space discretization, a 6th order scheme is employed to differentiate the diffusive term, whereas a 5th is applied for the convective term. A 4th order Runge-Kutta scheme is used to advance in time.

\subsection{Computational Setup}

A direct numerical simulation (DNS) of a subsonic round jet impinging on a semicylindrical concave wall is performed using the finite difference code developed in-house at the CFD Group of TU Berlin. The computational domain, sketched in Fig. 1 and consisting of a sector of a cylindrical circular shell, is discretized on a grid with resolution $1024 \times 512 \times 512$ in the azimuthal, radial and longitudinal directions, respectively. Two walls are located at the curved boundaries; the jet issues from an nozzle in the uppermost wall and impinges on the lowermost (target plate), whose relative curvature ${ }^{2}$ is 0.125 . The grid is refined around the jet axis and in proximity of the wall, leading to a maximum variation of cell spacing less than $1 \%$ in all directions and ensuring thereby a maximum value of dimensionless wall distance $y^{+}$at the closest grid points to the walls less than 0.6.

The jet Reynolds number (based on the nozzle diameter $D$ and the inlet bulk velocity $U_{b}$ ) and Prandtl number are set to 3300 and 0.71 , respectively. The ratio between the jet pressure at the inlet and the initial ambient pressure is chosen in order to ensure a fully expanded Mach number -equal to 0.8 . The initial ambient temperature equals the temperature of the target plate, which is kept uniform and constant; the initial temperature of the jet is also constant and $80 \mathrm{~K}$ below the temperature of the plate. A laminar inlet condition is enforced by using a standard hyperbolic tangent profile.

\footnotetext{
${ }^{2}$ The relative curvature of the target plate is defined as the ratio between the nozzle diameter and the radius of curvature of the plate.
} 
Fig. 1 Sketch of the computational domain. Walls are colored in grey, whereas inlet (orifice in the uppermost wall) and outlet are transparent. Lines A and $B$ will be used to present results on the curved and plane side of the surface, respectively. D indicates the orifice diameter (i.e. jet diameter at the inlet)

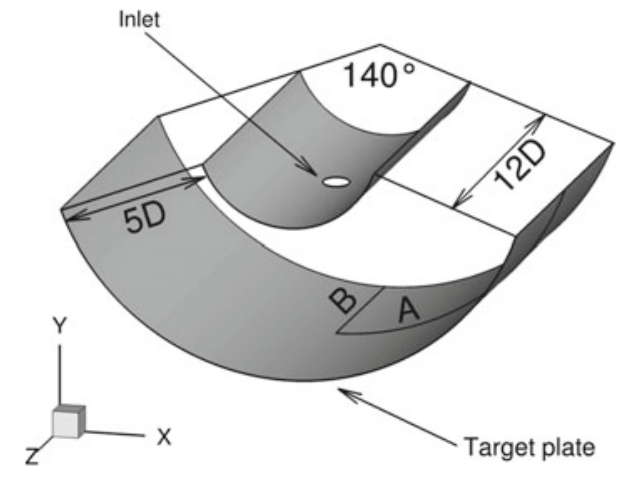

The choice of a laminar inlet ensures that spurious frequencies are not artificially inserted into the domain [16]. In the first stage of the computation, a thin annular disturbance is applied to the inlet profiles in order to facilitate the turbulent transition.

Non-slip conditions are enforced at the walls, whereas non-reflecting boundaries are used for the outlet. Furthermore, in order to destroy the vortices leaving the domain, a sponge region is implemented for $r / D>5$, with $r$ the distance from the jet axis. Namely, forcing terms are added to the right-hand side of the Navier-Stokes equations with magnitude proportional to the difference between the computed and reference quantities, which were evaluated preliminary through a large eddy simulation (LES) performed on a wider domain. It is worth noting that the data computed within the sponge region are disregarded as far as it concerns the discussion of results and the evaluation of statistics.

With the exception of the plate curvature, analogous numerical and physical parameters are employed in the works by Wilke and Sesterhenn [16-18], who showed its validity as a DNS study. Since the grid spacing is, in the present setup, nearly equivalent and the curvature is not deemed to affect noticeably the Kolmogorov microscales (i.e. the smallest scales of turbulence), the validity of the present study can be also ensured.

\section{Results and Discussion}

In the following, results of the calculation will be shown. Statistics were collected for a time equal to approximately $350 t_{r}$, where the reference time $t_{r}$ is given by $D / U_{b}$. This amount of time, corresponding to about 70 times as long as it takes the flow to reach the plate from the inlet, is deemed sufficient for the convergence of first statistical moments, here presented. Since the considered geometry is not axisymmetric, the data cannot be averaged over any statistically homogeneous direction. As shown 
Fig. 2 Averaged local Nusselt number $N u$ on the target plate as a function of the non-dimensional distance from jet axis $r / D$. Flat plate data courtesy of Wilke and Sesterhenn [16-18]

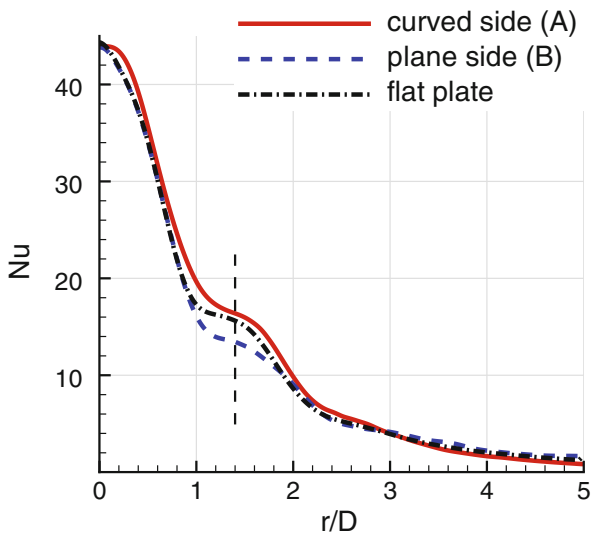

in Fig. 1 results will be therefore presented along two ${ }^{3}$ characteristic lines, A and B, respectively referred to as curved side and plane side.

The heat transfer intensity at the target plate may be expressed through the dimensionless Nusselt number, defined as:

$$
N u=\frac{\dot{q} D}{\lambda \Delta T},
$$

being $\dot{q}$ the heat flux, $\lambda$ the thermal conductivity of the fluid and $\Delta T$ the difference between the temperature of the isothermal plate and the bulk temperature of the jet at the inlet. Figure 2 compares the heat transfer calculated in the present case with that presented by Wilke and Sesterhenn [16-18], in the following referred to as reference or flat plate case. As already mentioned, Wilke and Sesterhenn studied indeed the heat transfer of a jet impinging on a flat plate under analogous conditions (e.g. Reynolds and Mach numbers, nozzle-to-plate distance, velocity and temperature inlet profiles, etc.). It may be observed that $N u$ on the curved side is everywhere higher than on the plane side and flat plate for dimensionless distances from the jet axis $r / D \lesssim 3$, beyond which it remains below the other curves. Furthermore, the slope of the flat plate curve at the jet axis is the lowest, whereas the plane side and flat plate curves exhibit similar behavior, resulting in a total heat transfer in this region higher than for the reference case. An inflection point, which replaces the characteristic secondary peak appearing for lower nozzle-to-plate distances [3], is also observable in all the curves. It is located on both the curved and plane sides at $r / D \simeq 1.4$, at an advanced location in comparison to the reference case where it was found at $r / D \simeq 1.2$. Elsewhere, the Nusselt number distribution follows more closely the reference case: at $r / D \gtrsim 2.5$ there is no visible difference when compared

\footnotetext{
${ }^{3}$ On the curved side of the impingement plate, $r / D$ is computed as the length of the arc with origin in the jet axis and running on the surface along line A. Negligible differences appear when computing $r / D$ as the Cartesian distance from the jet axis.
} 
Fig. 3 Instantaneous contours of $\mathrm{Nu}$ on the target plate. At the top-right corner a $3 \mathrm{D}$ rendering, which additionally shows the wall curvature, is plotted

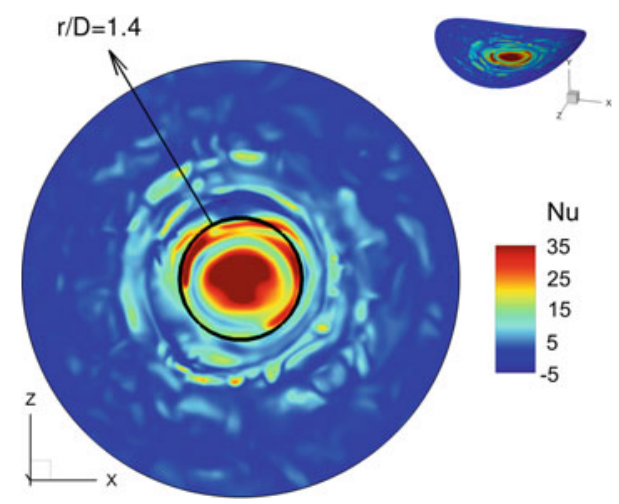

with both the curved and plane sides. The average Nusselt number within a circle of radius $r / D<5$ differs from the reference case by just $0.03 \%$. Consequently, it may be concluded that the integral heat transfer is not noticeably affected by the plate curvature.

The instantaneous heat transfer follows closely the evolution of turbulent structures at the wall, as shown in Fig. 3 through a snapshot of the instantaneous contours of $\mathrm{Nu}$ at the target plate. The highest heat flux occurs in a region around the plate center, where the cold jet core impacts the wall; thus, this region fluctuates as the jet core does in proximity of the impingement point. From this region, a series of annuli, characterized by high $\mathrm{Nu}$, travels away from the jet axis in a radial direction. At a certain distance from the centre $(r / D \gtrsim 2)$, each annulus loses symmetry while decreasing in intensity. The annuli are in fact directly related to the secondary vortices which originates in the wall jet region ${ }^{4}$ and are deemed responsible for the inflection point (or secondary peak) in the average $N u$ curve [16-18]. Interestingly, narrow zones of reverse heat transfer appear between two following annuli; as a matter of fact, within these regions of negative $N u$ the flow is cooled down by the plate. This phenomenon, counterintuitive at first glance, can be easily seen as an effect of fluid compressibility, ${ }^{5}$ friction and injection into the jet of hot fluid from the surrounding environment; indeed, all these physical mechanisms contribute to increase the fluid temperature, to such extent that in some regions, where reverse heat transfer occurs, it exceeds the wall temperature. Nevertheless, zones of reverse heat transfer are not observable on the average.

Figure $4 \mathrm{a}$ shows the time evolution of $N u$ at $r / D=1.4$ on both the curved and plane sides of the plate, where the inflection point appears. The oscillatory trend of $\mathrm{Nu}$ confirms the motion of the high heat flux annuli on the wall. Between two successive annuli, zones of low heat flux, characterized at certain times by the aforementioned reverse heat transfer, are observable. Within the considered time interval, peaks of

\footnotetext{
${ }^{4}$ On the other hand, primary vortices appear in the free jet region.

${ }^{5}$ In order to recognize the relevance of fluid compressibility effects to the case in analysis, it is worth recalling that the jet fully expanded Mach number is equal to 0.8 .
} 
similar intensity recurs on the curved side, whereas peaks on the plane side exhibit at first lower and then higher intensity.

In order to gain a better insight into the oscillatory nature of the heat transfer, a discrete Fourier transform of the instantaneous $N u$ signal at specific locations was performed. To this end, 1822 snapshots, covering a total time interval of approximately $66 t_{r}$, were used. Two different locations were selected, $r / D=1.2$ and 1.4 , representing the locations of the inflection points in the reference and present case, respectively. By analyzing Fig. 4b, it can be seen that no noticeable difference between the two chosen locations exists. Moreover, two peaks are clearly visible: the first at $S t=0.33$ and the second, of greater intensity, at $S t=0.62$ (the Strouhal number $S t$ is the dimensionless frequency, given by $f D / U_{b}$, with $f$ the frequency). Differently, peaks of the frequency spectrum were found in the reference case at $S t=0.46$ and 0.92 [17], i.e. at frequencies respectively $40 \%$ and $50 \%$ higher than in the present case. This result indicates that the introduction of a generic curvature in the impingement plate might determine a shift in the peak frequencies of some instantaneous fluid properties.

A Dynamic Mode Decomposition (DMD) of the flow was performed in order to analyze the turbulent structures responsible for the heat transfer at the main frequencies found through the Fourier analysis. The same time-window length and number of samples were used (see above). This method, first introduced by [19], decomposes the flow field as a superposition of modes. It is applicable even when the dynamics of the system is nonlinear and consists in extracting DMD modes and eigenvalues from a time series of collected data. The modes are spatial fields that usually identify coherent structure in the flow, whereas the eigenvalues represent, among other things, the oscillation frequency of each mode. As a result, both modes and

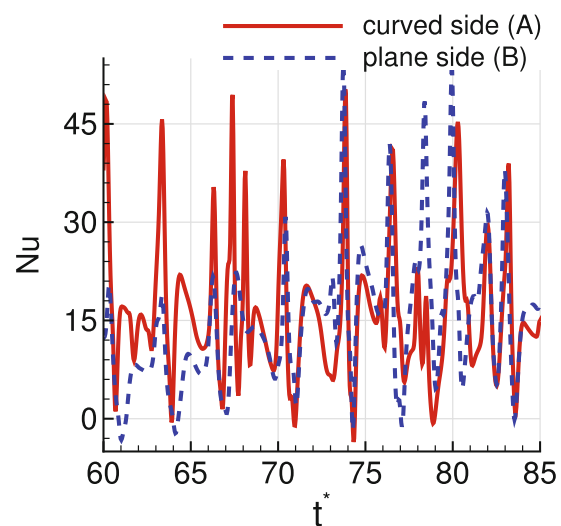

(a) Local instantaneous $\mathrm{Nu}$

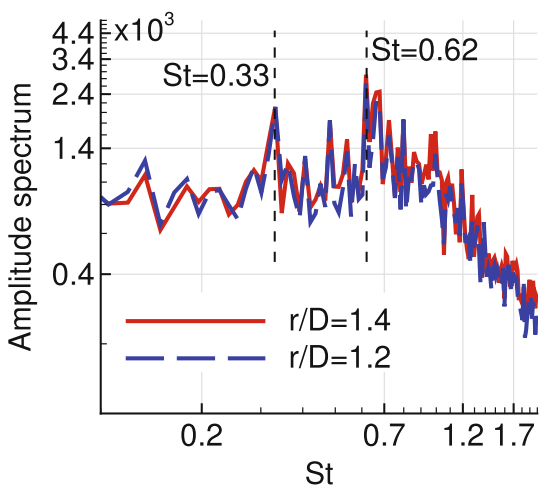

(b) Fourier transform of $N u$

Fig. 4 Local instantaneous Nusselt number at $r / D=1.4$ versus dimensionless time $t^{\star}=t / t_{r}$ a and amplitude spectrum of its Fourier transform computed at different radii versus Strouhal number b Please note that the Fourier spectrum in figure is the average between the spectra computed on both sides (curved and plane) at the indicated $r / D$ 


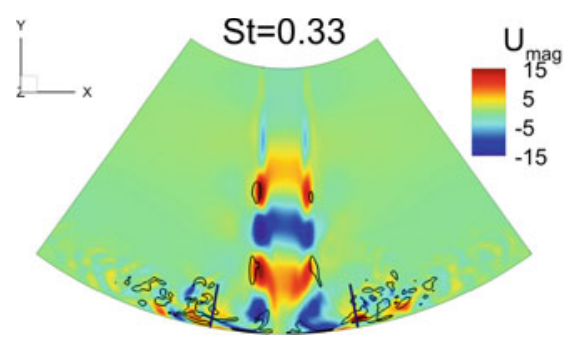

(a) $S t=0.33$

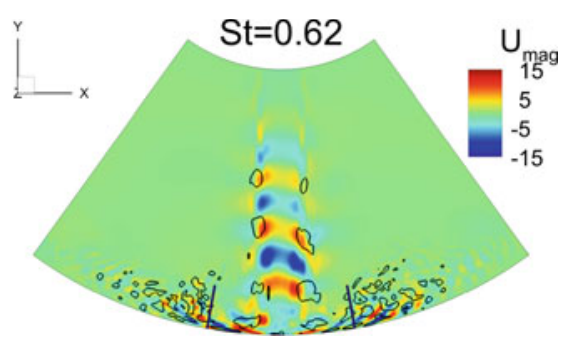

(b) $S t=0.62$

Fig. 5 Snapshots of DMD-reconstructed fields associated with different modes on a plane $x y$ passing through the jet axis, colored by the velocity magnitude and with isolines of $Q$, i.e. the second invariant of the velocity gradient tensor. The blue lines orthogonal to the wall indicate the position where $r / D=1.4$

eigenvalues describe the dynamic of an oscillatory flow field [21]. Once the DMD is performed, it is possible to reconstruct the field associated with a specific mode from the frequency back to the time domain. Two snapshots of the reconstructed velocity magnitude ${ }^{6}$ associated with the modes oscillating at $S t=0.33$ and 0.62 are shown in Fig. 5a, b respectively. The turbulent structures formed within shear layer of the free jet region are decomposed into two distinct structures; the largest of those are associated with the lowest frequency $(S t=0.33)$, whereas the smallest oscillates at the highest frequency $(S t=0.62)$. In either case, the structures impinge on the wall and travel outward radially. In this stage, the largest structures lose intensity because of the impact with the target plate, whereas the intensity of the smallest enhances. This causes $N u$ to oscillate at $S t=0.33$ with an intensity lower than at $S t=0.62$ (see Fig. 4b). This behavior appears likewise in the refrence case, with the only, not negligible, difference in the magnitude of the characteristic frequencies.

Figure 6 shows the contours of the absolute values of the density gradient at four time instants uniformly spaced within a period associated with $S t=0.33$, with the last instant corresponding to the beginning of the following period. The snapshots highlight the life cycle of a typical Kelvin-Helmholtz instability, initially originated within the shear layer of the free jet region at about $1.5 \mathrm{D}$ from the nozzle exit; traveling downwards, the same instability rolls up and grows in size, until when, in the fourth snapshot, it loses symmetry, being stretched in the vertical direction. Within the same period, roughly two of those instabilities are transported towards the wall, in the proximity of which they break down into secondary vortices. It follows that the period corresponding to $S t=0.33$ is needed by single vortices to form, travel towards the wall and break, whereas the frequency corresponding to $S t=0.62$ (roughly double than the first) is associated with the generation of secondary vorticity in the wall jet region.

\footnotetext{
${ }^{6}$ Note that the reconstructed velocity magnitude can be negative, since it represents the portion of the velocity magnitude that oscillates with the frequency corresponding to the extracted mode.
} 


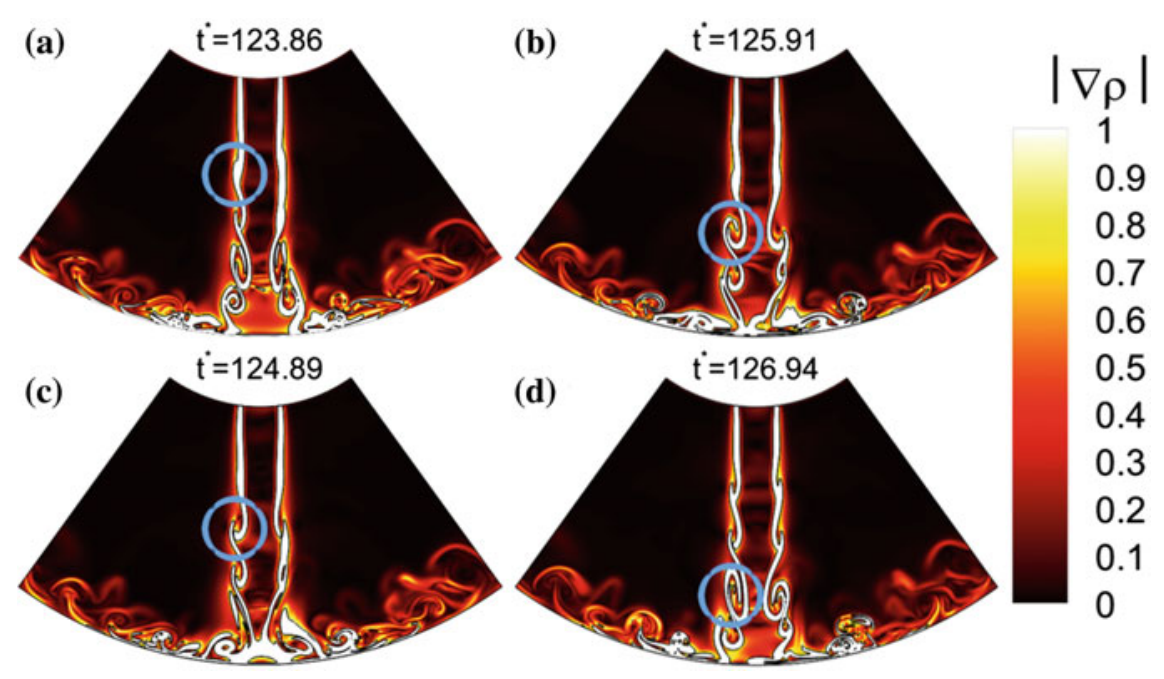

Fig. 6 Absolute value of density gradient on a plane $x y$ passing through the jet axis at four time instants uniformly spaced within the period $t_{r} / S t$, with $S t=0.33$. The dimensionless time $t^{\star}$ is given by $t / t_{r}$.

\section{Conclusions}

A round jet impinging on a curved concave surface has been investigated by means of a direct numerical simulation (DNS). The use of this method in a compressible case represents a novelty in the research field, especially in view of the interesting implications on the development of application oriented technologies, such as the internal cooling of gas turbine components. Results have been compared with those presented by Wilke and Sesterhenn [16-18], who studied a jet impinging on a flat surface under otherwise equivalent conditions.

Due to the low Reynolds number (3300), the high plate-to-nozzle distance $(5 \mathrm{D})$ and the laminar inlet condition, the characteristic secondary peak in the average Nusselt number profile at the target plate could not be observed. On the contrary, an inflection point appears. This latter is located at $r / D=1.4$, whereas Wilke and Sesterhenn found it at $r / D=1.2$. Despite this difference, the average heat flux integrated over the surface in $r / D<5$ does not differ in any significant manner.

The Fourier transform and dynamic mode decomposition (DMD) here performed showed, on the other hand, different constituent frequencies in the heat transfer. Indeed, the frequencies governing the generation, transport and breakup of the turbulent structures responsible for the heat transfer were found approximately $30 \%$ lower than in the case of jet impinging on a flat plate [17]. Two dominant frequencies have been observed: the lowest $(S t=0.33)$ being related with the period needed by a typical Kelvin-Helmholtz instability to be transported in the proximity of the target plate, the highest $(S t=0.62)$ governing instead the formation of secondary vorticity in the wall jet region. 
This indicates that the dynamic response of the impinging jet flow is affected by the curvature of the target plate, which cannot be therefore disregarded in the implementation of dynamic techniques for heat transfer enhancement, such as pulsating impingement cooling [22]. On the other hand, it is legitimate, for the analyzed geometry, to approximate curved surfaces with flat when time averaged quantities are sought.

It is finally worth noting that, in spite of the interesting results observed, the physics behind the frequency-shift remains to be fully explained. To this end, future work shall address the fluid dynamic stability of the system. This will allow the study of the receptivity of a range of different parameters on the system at acceptable computational cost.

Acknowledgements The authors gratefully acknowledge support by the Deutsche Forschungsgemeinschaft (DFG) as part of collaborative research center SFB 1029 "Substantial efficiency increase in gas turbines through direct use of coupled unsteady combustion and flow dynamics" on project B04.

\section{References}

1. Zuckerman, N., Lior, N.: Jet impingement heat transfer: physics, correlations, and numerical modeling. Adv. Heat Transf. 39, 565-631 (2006)

2. Hadžiabdić, M., Hanjalić, K.: Vortical structures and heat transfer in a round impinging jet. J. Fluid Mech. 596, 221-260 (2008)

3. Hattori, H., Nagano, Y.: Direct numerical simulation of turbulent heat transfer in plane impinging jet. Int. J. Heat Fluid Flow 25(5), 749-758 (2004)

4. Cornaro, C., Fleischer, A., Goldstein, R.: Flow visualization of a round jet impinging on cylindrical surfaces. Exp. Therm. Fluid Sci. 20(2), 66-78 (1999)

5. Lee, D., Chung, Y., Won, S.: Technical note the effect of concave surface curvature on heat transfer from a fully developed round impinging jet. Int. J. Heat Mass Transf. 42(13), 24892497 (1999)

6. Viskanta, R.: Heat transfer to impinging isothermal gas and flame jets. Exp. Therm. Fluid Sci. 6(2), 111-134 (1993)

7. Choi, M., Yoo, H.S., Yang, G., Lee, J.S., Sohn, D.K.: Measurements of impinging jet flow and heat transfer on a semi-circular concave surface. Int. J. Heat Mass Transf. 43(10), 1811-1822 (2000)

8. Gilard, V., Brizzi, L.E.: Slot jet impinging on a concave curved wall. J. Fluids Eng. 127(3), 595-603 (2005)

9. Hashiehbaf, A., Baramade, A., Agrawal, A., Romano, G.: Experimental investigation on an axisymmetric turbulent jet impinging on a concave surface. Int. J. Heat Fluid Flow 53, 167-182 (2015)

10. Yang, G., Choi, M., Lee, J.S.: An experimental study of slot jet impingement cooling on concave surface: effects of nozzle configuration and curvature. Int. J. Heat Mass Transf. 42(12), 21992209 (1999)

11. Aillaud, P., Duchaine, F., Gicquel, L.: LES of a round impinging jet: investigation of the link between Nusselt secondary peak and near-wall vorical structures. In: ASME Turbo Expo 2016: Turbomachinery Technical Conference and Exposition, vol. 5B: Heat Transfer, p. V05BT11A002. American Society of Mechanical Engineers (2016)

12. Jefferson-Loveday, R., Tucker, P.: LES of impingement heat transfer on a concave surface. Numer. Heat Transf. Part A Appl. 58(4), 247-271 (2010) 
13. Borello, D., Salvagni, A., Hanjalić, K.: Effects of rotation on flow in an asymmetric ribroughened duct: LES study. Int. J. Heat Fluid Flow 55, 104-119 (2015a)

14. Borello, D., Salvagni, A., Rispoli, F., Hanjalic, K.: LES of the flow in a rotating rib-roughened duct. In: Direct and Large-Eddy Simulation IX, pp. 283-288. Springer (2015b)

15. Borello, D., Rispoli, F., Properzi, E., Salvagni, A.: LES-based assessment of rotation-sensitized turbulence models for prediction of heat transfer in internal cooling channels of turbine blades. In: ASME Turbo Expo 2016: Turbomachinery Technical Conference and Exposition, vol. 5B: Heat Transfer, p. V05BT11A012. American Society of Mechanical Engineers (2016)

16. Wilke, R., Sesterhenn, J.: Numerical simulation of impinging jets. In: High Performance Computing in Science and Engineering vol. 14, pp. 275-287. Springer (2015)

17. Wilke, R., Sesterhenn, J.: Numerical simulation of subsonic and supersonic impinging jets. In: High Performance Computing in Science and Engineering vol. 15, pp. 349-369. Springer (2016)

18. Wilke, R., Sesterhenn, J.: Statistics of fully turbulent impinging jets. J. Fluid Mech. 825, 795824 (2017)

19. Schmid, P.J.: Dynamic mode decomposition of numerical and experimental data. J. Fluid Mech. 656, 5-28 (2010)

20. Sesterhenn, J.: A characteristic-type formulation of the Navier-Stokes equations for high order upwind schemes. Comput. Fluids 30(1), 37-67 (2000)

21. Tu, J.H., Rowley, C.W., Luchtenburg, D.M., Brunton, S.L., Kutz, J.N.: On dynamic mode decomposition: theory and applications (2013). arXiv preprint arXiv:1312.0041

22. Janetzke, T., Nitsche, W.: Time resolved investigations on flow field and quasi wall shear stress of an impingement configuration with pulsating jets by means of high speed PIV and a surface hot wire array. Int. J. Heat Fluid Flow 30(5), 877-885 (2009) 